Histoire de l'éducation

LEDUCATHON

140-141| 2014

L'État et l'éducation en France. XIX ${ }^{\mathrm{e}}-\mathrm{XX}^{\mathrm{e}}$ siècles

\title{
Les statisticiens du ministère de l'Éducation nationale : évolutions d'un métier d'État (1957-2007)
}

The statisticians of French ministry of education: development of a state profession (1957-2007)

\section{Xavier Pons}

\section{OpenEdition \\ Journals}

Édition électronique

URL : https://journals.openedition.org/histoire-education/2823

DOI : 10.4000/histoire-education.2823

ISSN : 2102-5452

Éditeur

ENS Éditions

Édition imprimée

Date de publication : 31 août 2014

Pagination : 115-132

ISBN : 978-2-84788-649-8

ISSN : 0221-6280

\section{Référence électronique}

Xavier Pons, «Les statisticiens du ministère de l'Éducation nationale : évolutions d'un métier d'État (1957-2007) », Histoire de l'éducation [En ligne], 140-141 | 2014, mis en ligne le 31 août 2016, consulté le 20 mai 2021. URL : http://journals.openedition.org/histoire-education/2823 ; DOl : https://doi.org/ 10.4000/histoire-education.2823 


\title{
Les statisticiens du ministère de l'Éducation nationale : évolutions d'un métier d'État (1957-2007)
}

\author{
Xavier Pons
}

Contrairement à l'image souvent véhiculée selon laquelle un État central fort serait très tôt amené à produire des statistiques, l'histoire du ministère de l'Éducation nationale français montre que la centralisation de cette production est récente $^{1}$. Si de premières enquêtes sont lancées dès 1797, il faut attendre 1957 pour que soit créé au sein de l'administration centrale du ministère un service statistique à part entière ${ }^{2}$. Pendant cinquante ans, ce service - qui devient une direction d'administration centrale en 1987 - met en œuvre différents outils statistiques permettant d'informer la politique scolaire d'État. Plusieurs missions lui sont assignées tout au long de son histoire : centralisation puis gestion de l'appareil statistique du ministère ${ }^{3}$, évaluation du système éducatif, prospective et depuis 2006, contribution à l'analyse de la performance de l'action de l'État. Cependant, derrière les apparences - le caractère cumulatif des travaux statistiques et l'enchaînement vertueux des nouvelles techniques

1 De nombreux éléments factuels à l'origine de cet article ont déjà été exposés en anglais, selon une approche théorique différente, dans une contribution au volume $12, \mathrm{n}^{\circ} 1$, de la revue European Educational Research Journal.

2 Jean-Noël Luc, La statistique de l'enseignement primaire. 19e-20 siècles. Politique et mode d'emploi, Paris, INRP/Economica, 1985, 242 p.

3 Plusieurs bureaux au sein de cette administration - leur liste précise varie selon les périodes - composent le "service statistique ministériel" de l'Éducation nationale et participent ainsi du "service statistique public" coordonné par l'Institut national des statistiques et des études économiques (INSEE) selon les termes de la Loi n $51-711$ du 7 juin 1951, plusieurs fois modifiée. 
d'enquête -, le métier de statisticien ministériel a en fait profondément évolué en cinquante ans, donnant naissance à un univers bureaucratique fortement clivé. Comment caractériser les transformations de ce métier tout au long de la période?

Notre hypothèse de départ est que ce métier n'évolue pas uniquement en fonction d'impératifs cognitifs (comment produire depuis une administration d'État une connaissance la mieux fondée possible?) qui eux-mêmes dépendraient en partie de l'évolution de disciplines académiques (mathématiques, statistique, économie, etc.) et des technologies disponibles (mécanisation, informatisation, multimédia); mais aussi en fonction des enjeux bureaucratiques et politiques du moment (conquérir, défendre et promouvoir une compétence au sein de l'administration en tenant compte des besoins des décideurs). Notre seconde hypothèse est que les clivages au sein du groupe professionnel que constituent les statisticiens du ministère de l'Éducation nationale se sédimentent dans le temps plus qu'ils ne se succèdent et qu'ils peuvent ainsi être réactivés, en des termes nouveaux, à différentes périodes.

Cet article analyse ainsi, sur la base de différents matériaux présentés dans une partie méthodologique liminaire, l'évolution de l'organisation de la production statistique, des techniques et des méthodes utilisées, de la composition socio-professionnelle des statisticiens et des principaux clivages qui les opposent. La conjonction de ces quatre variables met en lumière une périodisation spécifique, mais aussi des configurations sociales ${ }^{4}$ différentes d'exercice du métier de statisticien ministériel, desquelles émergent des figures ${ }^{5}$ historiques de ce dernier.

\section{Précisions méthodologiques}

Cet article se fonde sur des matériaux recueillis au cours d'une thèse de doctorat de science politique sur l'évaluation des politiques éducatives en France ${ }^{6}$. Si ce travail ne portait pas d'emblée sur le métier de statisticien, l'enquête a

\footnotetext{
4 Au sens de Norbert Elias, Qu'est-ce que la sociologie?, Paris, Agora Pocket, 1991, 222 p.

5 Ce terme ne désigne pas ici une personnalité marquante mais une forme, emblématique d'une période, prise par le métier de statisticien, donc le produit d'une configuration sociale et historique donnée.

6 Xavier Pons, L'évaluation des politiques éducatives et ses professionnels. Les discours et les méthodes (1958-2008), Paris, Institut d'études politiques, 2008, 961 p.
} 
révélé que nous ne pouvions pas comprendre les méthodes et les conceptions de l'évaluation mises en avant par les évaluateurs sans analyser les fondements de leurs identités professionnelles. Celles-ci étant fortement déterminées par la définition de leurs métiers initiaux sur lesquels est venue s'adosser leur mission nouvelle d'évaluation (métiers d'inspecteur, de statisticien, de chercheur, etc.), nous avons dû multiplier les méthodes de recherche pour comprendre leurs fondements historiques et leurs ressorts profonds.

Dans le cas des statisticiens, nous avons rassemblé de nombreux documents internes à l'actuelle Direction de l'évaluation, de la prospective et de la performance (DEPP), qui a repris en 1987, sous l'appellation de Direction de l'évaluation et de la prospective (DEP), les activités du service central des statistiques du ministère (notes internes, mémoires professionnels, programmes de travail annuel), ainsi que la littérature que ses membres ont pu éditer sur leur propre histoire ${ }^{7}$ et une archive nationale précieuse pour comprendre la période $1957-1965^{8}$.

Nous avons eu recours également à 32 entretiens effectués auprès de statisticiens ou de leurs dirigeants : 4 furent consultés au Service d'histoire de l'éducation de l'Institut national de la recherche pédagogique (INRP' ${ }^{9}$, archives orales sur les "Témoins et acteurs des politiques d'éducation depuis la Libération") et 28 furent réalisés auprès de membres passés et présents de la DEPP.

Enfin, la sociologie de ces membres se fonde sur l'analyse des 135 réponses à un questionnaire adressé en 2007 aux 502 chargés d'études ayant travaillé à la DEP(P) entre 1992 et 2007 et sur l'exploitation des annuaires du ministère. Les annuaires de l'Éducation nationale (années 1960, 1962, 1965, 1968, 1970 à 1975) et les annuaires détaillés de l'administration centrale du ministère (années 1976 à 1992) furent consultés à la mission des archives du ministère, 101 rue de Grenelle à Paris. Les annuaires de la DEP(P) furent obtenus directement auprès de la direction, pour ceux qui étaient encore en sa possession (années 1993 à 1998, 2001, 2002, 2004, 2006, 2007). Si ces

7 INSEE, Pour une histoire de la statistique, Paris, INSEE, t. 1, 1977, 593 p.; INSEE, Pour une histoire de la statistique, Paris, INSEE, t. 2, 1987, 969 p.; Pierre Fallourd, Annie Deruelle, Raymond Pelle, Système d'information et planification de l'éducation en France. t. 1, Paris, MEN, 1990, Rapport non publié remis à l'IIPE de l'UNESCO; DEP, Le pilotage du système éducatif. Enjeux, outils, perspectives, Paris, DEP-MENSR, 2004, 122 p.

8 M. Bertrand, M. Febvay, M. Kahn (1965), Rapport sur l'information statistique au Ministère de l'Éducation nationale, rapport du Comité national d'enquête sur le coût et le rendement des services publics, mars 1965, 70 p., AN, 19790742, art. 17, rapport n² 271.

9 Aujourd'hui remplacé par l'Institut français de l'Éducation (ENS de Lyon). 
annuaires constituent une source incomplète, ils contiennent des informations utiles sur le statut administratif des personnels, dont on peut déduire, à l'aide du questionnaire et des entretiens, des trajectoires professionnelles typiques ${ }^{10}$.

\section{Centraliser et produire : administrateurs et modernisateurs de la statistique (1957-1972)}

Au début de l'année 1957, la production statistique évolue selon des intérêts dispersés, au sein d'au moins quatre organismes différents : le Bureau universitaire des statistiques, le Centre d'études et de recherches documentaires sur l'enseignement technique, le bureau statistique de la direction de l'enseignement secondaire du ministère et l'Institut national d'études démographiques. Du fait des premiers signes de "l'explosion scolaire", le besoin de statistiques et de prévisions sur les effectifs d'enseignants et d'établissements s'accroît. Un arrêté ministériel du 27 mars 1957 crée, sur la base du bureau statistique de la direction du second degré, le "Service technique de statistique scolaire». L'enjeu est alors de centraliser la production de données au sein du ministère dans un contexte où "les bureaux compétents se [préoccupent] de dresser, chacun selon sa méthode, les statistiques des établissements qu'ils [ont] la charge d'administrer" ${ }^{11}$. Aussi le service intègre-t-il progressivement entre 1958 et 1963 les statistiques sur les enseignements primaire et technique. En novembre 1963, il fait partie d'un nouveau secrétariat général supposé coordonner les activités des directions du ministère. Son chef, Pierre Laurent, insiste plusieurs fois dans ses discours et notes de service sur la nécessaire centralisation des données et sur le monopole de l'usage du terme "statistique», réservé au Service central des statistiques et sondages (SCSS) tout juste créé en mars $1964^{12}$.

Toutefois, cette centralisation reste inachevée en 1965. Les organismes mentionnés plus haut continuent à publier des statistiques sur le système scolaire. La centralisation des données n'est effective que pour les questionnaires d'établissements du second degré. Pour le premier degré, elle n'est réalisée qu'au niveau du département, le ministère ne centralisant que les synthèses

10 Pour plus de précisions méthodologiques, voir notre thèse (p. 895-961).

11 AN, 19790742, p. 6.

12 Philippe Bongrand, Je t'aime... moi non plus. Éléments pour une histoire des rapports entre la réforme de l'enseignement et les sciences humaines et sociales 1959-1968, Paris, Rapport à la Fondation Charles de Gaulle, Juin 2007, 73 p. 
des inspecteurs d'académie. Or celles-ci sont souvent courtes, générales, et de qualité inégale, les inspecteurs d'académie n'ayant en général ni le temps ni les moyens humains et techniques pour s'assurer de la généralité, de la solidité et de la cohérence des données recueillies. Par ailleurs, quand les interlocuteurs de l'administration du ministère (cabinet, Direction de l'aménagement du territoire, ministère de l'Économie et des Finances, Plan) ont besoin de données chiffrées, ils tendent à passer directement par les directions concernées et non par le service statistique compétent ${ }^{13}$, quand ils ne produisent pas eux-mêmes leurs données en s'adressant directement aux inspecteurs d'académie ${ }^{14}$.

Néanmoins, à partir de 1967, le développement de la mécanographie et l'acquisition d'ordinateurs IBM $^{15}$ permettent au service d'augmenter et de diversifier sa production ${ }^{16}$. Auparavant, la statistique produite par le ministère était essentiellement descriptive et correspondait à "une activité administrative d'enregistrement de données diverses ${ }^{17}$. Elle reposait principalement sur l'enquête de rentrée annuelle (envoi aux directeurs d'établissement d'un questionnaire de plusieurs pages accompagné d'une circulaire ${ }^{18}$ exploitée de manière artisanale et elle concernait principalement les effectifs d'élèves et d'enseignants, les diplômes, et la situation matérielle et financière des établissements ${ }^{19}$. Désormais, les statisticiens du ministère peuvent traiter des échantillons de plus grande importance. Ils intègrent progressivement les enseignements de la théorie des sondages et des estimations : propriétés des estimateurs, plans de sondage, échantillons par quotas ou aléatoires. Dès 19641965, se développent les méthodes d'évaluation des effectifs attendus pour alimenter les projections du Plan sur les effectifs d'élèves et la construction d'établissements. Enfin, les progrès technologiques permettent de calculer plus finement les taux de scolarisation et de redoublement, et de constituer dès 1972 un panel d'élèves.

13 AN, 19790742.

14 Philippe Bongrand, Je t'aime... moi non plus..., op. cit.

15 International Business Machines.

16 Ils impliquent aussi pour le service d'être localisé dans de plus grands locaux à Vanves, au sud de Paris.

17 Alain Desrosières, La politique des grands nombres, Paris, La Découverte, 2000, p. 21.

18 AN, 19790742.

19 Jean-Pierre Briand, Jean-Michel Chapoulie, Henri Peretz, "Les statistiques scolaires comme représentation et comme activité", Revue française de sociologie, vol.20, 1979, p.669-702; Jean-Noël Luc, La statistique de l'enseignement primaire..., op. cit. 
Ces transformations organisationnelles et méthodologiques bouleversent sensiblement la composition du service statistique. Avant 1964, ce dernier est essentiellement composé d'une quarantaine d'agents qui dépendent de l'Institut pédagogique national (IPN). Ces "petites mains ${ }^{20}$ ont des statuts administratifs divers (agents ou assistants IPN, maîtres auxiliaires, dessinateurs, dactylographes). Leur travail concret consiste soit en des tâches purement administratives (secrétariat notamment), soit en des tâches de production toutes liées au support papier de l'époque (envoi des questionnaires par courrier, dénombrement et classement des fiches établissements, reprographie des enquêtes, présentation formelle des documents du ministère). Leur travail d'exécution est encadré par Raymond Pelle, ancien instituteur, ancien intendant et membre de l'administration centrale du ministère depuis 1953. Après 1964, cette composition évolue du fait de l'action de modernisateurs (par exemple Pierre Laurent, Gabriel Ducray, Alain Darbel, Maurice Febvay, etc.) qui, sous couvert d'un progrès technique nécessaire, s'appuient sur les recommandations du Commissariat général au Plan et sur leur expérience professionnelle (en général extérieure au secteur de l'éducation) pour restructurer le service statistique. L'impératif de faire passer ce dernier "d'un stade resté jusqu'ici artisanal à un stade industriel $\aleph^{21}$ et d'améliorer les prévisions d'effectifs implique un besoin croissant de personnes capables d'interpréter des données chiffrées. En conséquence, même si les effectifs globaux du service augmentent pendant la période (de 41 membres en 1963 à 96 en 1972), ses dirigeants se séparent progressivement entre 1964 et 1970 des administrateurs de la statistique pour recruter de plus en plus d'enseignants, de conseillers d'orientation et de contractuels ayant étudié les sciences sociales (sociologues, démographes, économistes). Les tout premiers administrateurs INSEE et ingénieurs informatiques arrivent dès 1969.

Ces évolutions suscitent différents clivages au sein du service. À ceux d'une organisation taylorienne classique, qui oppose une majorité de personnes exécutant des tâches répétitives à un encadrement limité ayant le monopole de l'activité intellectuelle de présentation des données, s'ajoute un clivage géographique. Les exécutants restent à Vanves, alors que les cadres sont plus fréquemment en relation avec l'administration centrale qui est rue de Grenelle à Paris. Ces

20 Entretien avec un chargé d'étude de la DEPP.

21 AN, 19790742, p. 2. 
derniers reprochent aux premiers de vivre en monde clos et de ne pas assez intégrer dans leurs pratiques professionnelles les impératifs, notamment gestionnaires, de la vie d'une administration. Ce clivage est redoublé d'un clivage politique. L'ouverture à de nouvelles catégories de personnels pratiquant les sciences sociales, qui ont tendance à se recruter par cooptation, et la persistance de plusieurs administrateurs peu qualifiés, se traduisent par le rassemblement au sein du service statistique d'individus de gauche dans un univers où les responsables administratifs, du fait de l'absence d'alternance politique, sont plutôt de droite. "Vanves" est ainsi fréquemment appelé le "Soviet" ou "les Rouges" par "la rue de Grenelle». Exemple typique de la conjonction de ces clivages, le service statistique se met en grève le 30 mai 1968 pour condamner la "déviation politique» dans l'usage de ses travaux. Les grévistes prétendent que le "service a été essentiellement utilisé pour cautionner une certaine politique [et plus] pour "justifier" des décisions déjà prises que pour préparer celles-ci". Ils regrettent que «les enquêtes statistiques [aient] été conçues dans une optique presqu'exclusive de gestion" et réclament que leur prise d'information soit fondée sur des "hypothèses de travail» claires, que le service ait plus de crédits pour financer des recherches académiques et que la statistique soit "reconnu[e] et défini[e] comme un instrument technique et scientifique $»^{22}$. La grève annonce en cela un clivage ultérieur entre les partisans de l'indépendance scientifique et ceux qui souhaitent plutôt accompagner une action politique.

\section{Rationaliser la production : les statisticiens de Vanves et les autres (1973-1987)}

À partir de 1973, l'organisation de la production statistique connait un premier mouvement de rationalisation. Le mandat du service ${ }^{23}$, tout comme sa position au sein du ministère ${ }^{24}$, sont plus précis. En termes de structures, le service est

22 Les citations sont tirées du texte adopté par l'assemblée générale du personnel en grève du 30 mai 1968 que nous nous sommes procuré au cours d'un entretien.

23 "Le [service statistique] est responsable de la production, du contrôle et de l'analyse des informations statistiques nécessaires à la conception et la mise en œuvre de la politique éducative d'une part, et de la gestion du système éducatif d'autre part. Il a la responsabilité de la politique des applications informatiques aux tâches de gestion du ministère; il doit également préparer un programme de modernisation de la gestion déconcentrée, enfin, mettre en œuvre des méthodes d'évaluation des résultats des actions d'éducation et de formation" (Annuaire de l'administration centrale, 1975, p. 67).

24 Après la disparition du secrétariat général et un éphémère passage sous l'égide d'une "Direction de la prévision" (1972-1973), le service devient un service central autonome se positionnant dans 
constitué entre 1973 et 1982 de quatre pôles majeurs qui confirment la tendance à la centralisation de la production statistique : un pôle chargé de gérer le système informatique du ministère, un pôle consacré à l'informatique de gestion, un pôle d'études lié à la rationalisation des choix budgétaires (RCB) et un pôle qui sera progressivement chargé de l'évaluation du système éducatif. Ces pôles intègrent plusieurs départements nouveaux au fur et à mesure de l'évolution de l'informatique de gestion et de la problématique de l'évaluation. Ils sont réorganisés en 1982, puis en 1985. Deux grandes sous-directions apparaissent clairement : une "sous-direction de l'évaluation du système éducatif" (SDEVA) et une "sous-direction des enquêtes statistiques et des études" (SDESE). Cette dernière, dirigée par un administrateur de l'INSEE, concentre en treize bureaux toutes les activités de production et d'exploitation des enquêtes statistiques.

En termes de gestion du personnel, les statuts administratifs des contractuels recrutés en urgence au cours de la période précédente pour faire face à l'impératif de production sont harmonisés. Claude Seibel, administrateur INSEE à la tête du service de 1973 à 1982, négocie avec cet institut le détachement de certains de ses personnels dans le service pour une période de trois ans. Leur nombre croît de façon régulière au cours de la période (de 3 en 1973 à 12 en 1987). À l'exception des administrateurs INSEE chefs de département, il s'agit principalement de jeunes femmes. Tous travaillent au sein de la SDESE, sauf Claude Seibel qui dirige le service et le département de l'évaluation. Enfin, selon ses besoins, le service statistique a recours aux compétences de quelques professeurs du secondaire et de conseillers d'orientation, dont certains viennent de l'Institut national d'étude du travail et d'orientation professionnelle (INETOP).

Mais c'est surtout en termes de méthodes et d'outils statistiques que les changements sont les plus probants. Deux panels d'élèves sont mis en œuvre en 1972-1974 et en 1978. Les informations recueillies permettent aux statisticiens d'étudier les effets d'actes plus pédagogiques comme le redoublement ${ }^{25}$ et de lancer les premières évaluations de masse des acquis des élèves dès 1974 (celles-ci ont lieu pratiquement chaque année à partir de 1979). En 1975, Denis Meuret, alors chargé d'études, met en place dans l'académie de Toulouse les premiers tableaux de bord des établissements qui sont généralisés en 1978 à

l'organigramme du ministère comme un interlocuteur des directions pédagogiques et du cabinet, au-dessous cependant des autres directions techniques.

25 Claude Seibel, "Genèses et conséquences de l'échec scolaire : vers une politique de prévention", Revue française de pédagogie, ${ }^{\circ} 67$, avril-mai-juin 1984, p.7-28. 
toutes les académies. En 1976, le service expérimente en collaboration avec les économistes de l'Institut de recherche sur l'économie de l'éducation (IREDU) le chiffrage d'un compte de l'éducation pour l'année 1971. En 1982, les premières enquêtes d'insertion sociale et professionnelle des élèves sept mois après leur sortie du système éducatif voient le jour. Elles sont généralisées à l'ensemble des académies en 1986. L'introduction de la micro-informatique au ministère en 1985 permet la création d'une première base informatique de gestion des élèves du second degré (Scolarité) ${ }^{26}$. En 1986, le service met au point, sur la base des matrices de flux calculées à la fin des années 1960, un premier modèle de prévision des effectifs (modèle BERSI) qui permet d'estimer les besoins en enseignants du second degré.

Toutefois cette rationalisation présente deux limites. D’une part, elle dépend grandement d'un contexte politique plus ou moins porteur, et de la capacité des statisticiens à traduire les impératifs de leur production conformément aux intérêts des décideurs du moment. Si le début des années 1970 constitue une période favorable (sensibilité au besoin de statistiques du ministre Joseph Fontanet, premières influences internationales et débuts de la RCB qui permet au ministère de recruter plusieurs chargés d'études formés aux sciences économiques), il faut toute l'ingéniosité de Claude Seibel pour que le soufflé ne retombe pas dès 1974. Ce dernier parvient à convaincre le ministre René Haby de l'utilité d'accompagner la réforme du collège unique par la mise en œuvre d'évaluations des acquis des élèves pour mesurer le degré de maitrise des nouveaux programmes. Aussi, après son départ, le service statistique se replie-t-il sur une activité essentiellement gestionnaire conformément aux commandes politiques qui lui sont adressées. La situation éminemment politique de 1986 relance les activités des statisticiens, ceux-ci étant incités à alimenter en projections statistiques ce qui deviendra le "Plan pour l'avenir de l'Éducation nationale" présenté le 15 décembre 1987 par René Monory. D’autre part, l'activité du service est fortement segmentée. La mise en place des nouveaux outils mentionnés plus haut implique pour chaque division de se spécialiser pour accumuler les données et les compétences nécessaires. Les chargés d'études du service ne savent pas toujours exactement ce que font leurs collègues émanant d'autres bureaux (certains ignorent par exemple que le ministère fait des

26 Pierre Fallourd, Annie Deruelle, Raymond Pelle, Système d'information et planification de l'éducation en France..., op. cit. 
études RCB) et le développement des premiers travaux d'évaluation se fait en marge de l'activité du service statistique de Vanves ${ }^{27}$.

Dans ce contexte, de nouveaux clivages apparaissent au sein du groupe professionnel. Le premier oppose les producteurs aux utilisateurs de données. À bien des égards, il est la nouvelle traduction du clivage taylorien de la période précédente entre exécutants et cadres administratifs, mais dans un contexte de développement des techniques statistiques. Les utilisateurs sont ceux qui exploitent les enquêtes. Ils ont généralement un profil sciences sociales au sens large, d'enseignants, de conseillers d'orientation ou d'administrateurs INSEE. Les producteurs prennent des visages différents selon les cas. Parfois il s'agit des ouvriers chargés de surveiller les outils mécanographiques, parfois des informaticiens responsables de l'informatique de gestion. Le plus souvent, il s'agit des administrateurs chargés de la saisie, de l'envoi des questionnaires, ou de l'impression des tableaux et graphiques. Les producteurs reprochent aux utilisateurs de ne pas s'abaisser à participer à la dure besogne de la production statistique (passation des questionnaires, constitution et de vérification des bases de données) et de s'en tenir à la partie noble et valorisée (exploiter les résultats de l'enquête et les publier dans une étude). Les utilisateurs prétendent que penser ainsi revient à ignorer la difficulté de l'interprétation des données. Ils s'indignent de ce que les producteurs de données usent toujours de cet argument pour justifier des augmentations de salaire ${ }^{28}$. Ils leur reprochent parfois de ne pas assez tenir compte dans la mise en œuvre de l'enquête statistique des impératifs de l'étude qui sera faite en aval de leur travail ${ }^{29}$. Un deuxième clivage oppose les défenseurs d'une statistique scientifique et les autres. Les premiers défendent une production rigoureuse et une interprétation objective

27 Xavier Pons, L'évaluation des politiques éducatives et ses professionnels..., op. cit.

28 "Il y a une grande ambivalence à l'égard de la fonction étude. D'un côté les gens qui ne font pas des études, la majorité, sont un peu envieux des gens qui font des études qui sont censés faire le travail intéressant alors qu'eux font le pénible et modeste travail de production, parce qu'ils n'ont jamais fait d'études en fait sinon ils verraient le côté déplaisant à faire des études. Donc il y a ça, et ils ont toujours tendance à ce que cela joue comme phénomène compensatoire pour leur carrière. Un tel fait des choses hyper intéressantes, il fait des études, moi j'ai les mains dans le cambouis, donc je suis plus méritant que lui si vous voulez" (entretien avec un chargé d'études de la DEPP).

29 "Jusqu'à ce moment-là [le milieu des années 1980], on était tributaire des informaticiens à qui on passait commande pour un tableau. Huit jours après on recevait le tableau : "ah non, ça nous plaît pas”. Et puis on renvoyait ou on laissait tomber de guerre lasse. Donc ça limitait toute inventivité dans les analyses qu'on pouvait faire. On était totalement limité à l'avance par les sorties qu'on pouvait faire d'une enquête. Donc en fait, c'était totalement routinisé. Point" (entretien avec un chargé d'études de la DEPP). 
des données. Cette dernière implique selon eux de prendre le temps de recouper et d'analyser les données et de ne pas s'aventurer dans des propos normatifs. Les seconds composent un groupe hétérogène. Il peut s'agir du producteur de données peu scrupuleux, du chef de service qui veut tordre les conclusions d'une enquête selon la modernité du moment ou du chargé d'études "politique". Le "politique", c'est celui qui doit sa place à des réseaux particuliers, celui qui comprend "trop" bien les impératifs politiques du cabinet en place ou celui qui n'ose pas défendre une vérité statistique déplaisante. Ce clivage recoupe parfois des considérations statutaires qui renvoient à des trajectoires professionnelles différentes (les "INSEE» d'un côté, les statisticiens du ministère au sens strict de l'autre). Il recoupe également le clivage entre les statisticiens de la "SDESE" et les membres de la "SDEVA». La position des premiers à l'égard de l'évaluation est ambiguë. Si cette dernière repose parfois sur des méthodes statistiques élaborées (usage de modèles psychométriques par exemple) et constitue en cela une activité noble, elle comporte un risque - celui d'associer le service à l'entreprise de modernisation de l'administration et à la prise de décision politique - et donne parfois lieu à des productions trop "littéraires". Une fois encore, la coupure géographique a pour effet de consacrer ces antagonismes. Enfin, les considérations politiques de la période précédente sont toujours présentes, qu'il s'agisse du clivage droite/gauche très prégnant au tout début des années 1980 ou de l'opposition aux "soixante-huitards" contestataires ${ }^{30}$.

\section{Produire pour tous : les statisticiens du miroir (1987-1997)}

Le mouvement de rationalisation de la période précédente aboutit en février 1987 à la création de la Direction de l'évaluation et de la prospective (DEP) ${ }^{31}$, qui s'impose comme un interlocuteur clé du cabinet et devient l'acteur central de

30 "Il y a quelque chose de fondamental dans l'histoire de notre génération, plus de gauche. C'est qu'on a avec $1981 \mathrm{vu}$ arriver dans des sphères de pouvoir des gens qu'on connaissait. [...] Donc cette sphère du pouvoir tout à coup devenait plus proche et devient des gens avec qui on pouvait parler. Par rapport à une période où on avait des gens totalement étrangers, totalement hostiles et d'un univers culturel totalement différent. Tout à coup, il n'y a plus cette barrière totale de génération et surtout d'univers culturel. [...] C'est des gens qui arrivent avec un autre background. Il y a plus d' "intellectuels". Il y avait plus de profs. Pour nous ça change la perception qu'on a des relations avec le pouvoir." (entretien avec un chargé d'études de la DEPP).

31 Décret 87-83 du 10 février 1987. 
la nouvelle politique d'évaluation lancée par le ministère en $1989^{32}$. Les statisticiens s'invitent dans de nouveaux débats sur le redoublement, le niveau des élèves, l'effet établissement ou l'effet de la réduction de la taille des classes. Ils représentent la France dans diverses organisations internationales ${ }^{33}$. À partir de 1990, la DEP a pour ambition d'être une interface légitime entre les décideurs politiques, l'administration du ministère, la recherche académique et les médias. La statistique et l'évaluation doivent contribuer à structurer le débat public et piloter le système éducatif en produisant "l'effet miroir " ${ }^{34}$. La DEP se lance alors dans une politique active de communication de ses travaux. La direction fait des conférences de presse après chaque enquête d'envergure. Elle édite plusieurs publications à grand tirage : les Notes d'information (une quarantaine par an en moyenne), les Dossiers de la DEP (entre 4 et 10 par an depuis le premier numéro de 1989), L'état de l'école lancé en 1991 (45 000 exemplaires par an) et Géographie de l'école lancée l'année suivante (même tirage). La DEP multiplie également les commandes effectuées auprès de chercheurs dans le double objectif de contrebalancer le poids des cabinets privés dans la part de son budget consacrée à ces commandes et de structurer la production de connaissances scientifiques sur le système éducatif. L'analyse de l'évolution de ce poste de dépenses depuis 1967 révèle que la période 1988-1997 est la seule où l'on voit augmenter en tendance et le volume global du budget (entre $463000 \mathrm{~F}$ et $1891000 \mathrm{~F}$ selon les années) et le nombre d'études (entre 17 et 43 selon les années) $)^{35}$.

Cette politique s'accompagne d'une hausse, sensible par rapport aux périodes antérieures, des effectifs de la direction qui se stabilisent à partir de 1992 autour de 220 personnes. Dans la mesure où celle-ci contrôle peu son recrutement (le nombre de postes ouverts étant décidé au niveau du ministère et en fonction des statuts administratifs en vigueur), la répartition des effectifs entre ces différents statuts est globalement stable. Toutefois, deux catégories

32 Xavier Pons, L'évaluation des politiques éducatives et ses professionnels..., op. cit.

33 La DEP participe activement à la création des indicateurs comparatifs sur l'enseignement de l'OCDE (projet INES) et représente la France à partir des années 1990 au sein de l'IEA (Association for the Evaluation of Educational Achievement).

34 Par cette expression, Claude Thélot (directeur de la DEP de 1990 à 1997) désigne dans plusieurs de ses publications la mise à disposition des acteurs du système éducatif de données sur les résultats de leur action visant à inciter ces derniers, face au miroir qui leur est tendu, à trouver d'eux-mêmes les remédiations nécessaires.

35 Xavier Pons, L'évaluation des politiques éducatives et ses professionnels..., op. cit. 
de personnel voient leur importance augmenter, confirmant ainsi un besoin accru de compétences techniques et scientifiques. Du fait de la titularisation de nombreux contractuels en 1990, la proportion d'ingénieurs d'étude ou de recherche augmente de 13,1\% en 1992 à 15,2\% en 1997. Au cours de la même période, celle des personnels de l'INSEE passe de 8,1\% à 11,4\%. Aux yeux de ces derniers, la DEP apparaît en effet comme un service statistique ministériel dynamique, compétent et représenté par un directeur sensible aux impératifs de la raison statistique, et par ailleurs issu du même institut.

D'un point de vue méthodologique, la DEP met en œuvre pendant cette période plusieurs outils statistiques en direction de l'administration et des enseignants (panel d'élèves en 1988, systématisation des évaluations CE2sixième à partir de 1989, chiffrage d'un compte de l'éducation en 1990 pour la période 1974-1988, base centrale de pilotage en 1991, évaluation diagnostique à l'entrée en seconde en 1992, généralisation du système Scolarité en 1993, indicateurs de valeur ajoutée des lycées en 1994), des usagers (publications, base centrale des établissements) et de la recherche (panels de 1989, 1995 et 1997, relance des évaluations bilans à partir de 1995), qui contribuent à asseoir sa réputation d'interlocuteur objectif et légitime. La mise en place d'applications Windows en 1991, le passage à la messagerie électronique pour tous en 1992 , la création en 1994 d'un Centre informatique d'aide à la décision (CISAD) et le développement des premières applications Web en 1995 améliorent par ailleurs l'efficacité et la coordination du travail des statisticiens. Du point de vue des techniques statistiques utilisées, le début des années 1990 est marqué par une mention plus fréquente, dans les publications, d'analyses de régression et d'analyses factorielles, même si la statistique produite reste essentiellement descriptive et vise à classer les phénomènes observés dans des catégories neutres utiles aux décideurs ${ }^{36}$.

Ce contexte fait évoluer les clivages internes au groupe professionnel. Les statisticiens sentent que leurs revendications méthodologiques sont prises en compte et même défendues auprès du cabinet. Les différents chantiers ouverts par la direction les stimulent et celle-ci gagne en notoriété. Ils ont l'impression de contribuer au débat public en produisant des constats scientifiques qui structurent ce dernier. En termes de clivages, il s'agit moins d'opposer de façon binaire des statisticiens scientifiques avant tout à des statisticiens 
plus politiques que de se positionner sur l'échelle du constat le plus juste. Un nouveau clivage apparaît ainsi entre, d'un côté, les personnels de l'INSEE en détachement pour trois ans (parfois plus dans le cas des chefs de bureau), de plus en plus nombreux et globalement mieux formés à la maîtrise des outils statistiques, et de l'autre, les statisticiens du ministère, notamment ceux de Vanves, qui sont en général en poste depuis plusieurs années et qui ont la mémoire de l'institution. En entretien, les premiers évoquent leurs difficultés à s'insérer dans un univers administratif qui est cloisonné, qui fonctionne sur le mode de l'évidence et où chaque bureau, voire chaque individu, a développé ses habitudes et ses pratiques statistiques qu'il suppose connues de tous, étant donné son ancienneté. Ils s'interrogent également sur la pertinence de certains choix méthodologiques et regrettent que leur plus-value en termes de statistiques soit parfois mal vécue par les seconds. Ces derniers, qui se sont habitués à voir venir des personnels INSEE voulant tout changer en trois ans avant de devoir repartir, insistent sur le fait qu'un constat objectif et utile au débat public ne se fonde pas que sur une technique statistique ardue mais suppose une connaissance fine de l'institution. La technique n'est qu'un moyen et non une fin en soi pour eux ${ }^{37}$. De même les clivages politiques évoluent. Il s'agit moins d'affirmer des convictions partisanes dans un contexte de cohabitations et de changements de plus en plus fréquents des équipes ministérielles que de veiller à ne pas faire de la DEP une agence de communication pour le ministre. C'est la crainte de certains statisticiens, de Vanves notamment, à l'arrivée de Claude Thélot. L'idée que l'évaluation et la production statistique doivent aider au débat public suscite quelques réticences et des craintes parmi les statisticiens soucieux avant tout du caractère objectif et scientifique de leurs travaux. Le clivage entre la SDESE et la SDEVA, entre une production scientifique autonome et une évaluation liée au politique, entre Vanves et la Rue de Grenelle s'en trouve réactivé. À chaque fois qu'ils présentent un nouvel outil statistique, les statisticiens doivent sans cesse faire la démonstration de leur autonomie scientifique auprès de leurs partenaires. Le clivage apparaît également quand il s'agit de gérer les publications des statisticiens. Celles-ci sont toutes soumises à l'aval de la hiérarchie, du chef de bureau jusqu'au directeur de cabinet. La présentation de certains chiffres ou la formulation de

37 "Les personnels INSEE, on a du mal à leur faire comprendre qu'il ne faut pas qu'ils s'enferment dans leur univers de savoir-faire. Or c'est facile car tout le monde a une expérience de l'école" (entretien avec un sous-directeur de la DEPP). 
certains constats sont parfois objets de négociations entre les chargés d'études et les différents niveaux hiérarchiques dont la volonté de défendre la raison statistique aux dépens des impératifs politiques est inégale selon les cas.

\section{Contrôler la production : la fin des statisticiens du miroir? (1997-2007)}

La dynamique impulsée par la DEP dans les années 1990 s'arrête avec l'arrivée de Claude Allègre à la tête du ministère en juin 1997. Ses positions scientifiques personnelles sur la statistique (pseudoscience non expérimentale, pour ce géochimiste de renom) et politiques sur l'évaluation (qu'il entend externaliser auprès d'une agence indépendante composée principalement de chercheurs sur le modèle du Conseil d'analyse économique créé par le Premier ministre en juillet 1997) le conduisent à profondément réorganiser la direction. Rebaptisée, celle-ci intègre une "sous-direction des constructions et du développement régional" inédite dans son histoire. L'évaluation ne disparait pas complètement mais est reléguée au rang de "mission" dont les activités doivent être programmées et encadrées par un "Conseil de l'évaluation" placé auprès du directeur et composé de chercheurs (comme Philippe Meirieu ou Christian Baudelot), sous la direction d'un inspecteur pédagogique régional. Au cours de la période suivante (2000-2006), les responsables de la direction s'attachent à la reconstruire et à revaloriser la mission d'évaluation. Il faut de nouveau publier, relancer les travaux avec les chercheurs, ouvrir l'accès aux données aux différents partenaires, raffermir les relations avec les autres directions. La DEP et la SDEVA reprennent en 2003 leur appellation d'origine. En 2004, la direction s'invente une tradition et célèbre, non sans reconstruire l'histoire, ses quarante ans d'existence ${ }^{38}$. À quelques exceptions près, les acteurs qui font l'évaluation du système éducatif depuis 30 ans sont invités à débattre du pilotage du système éducatif. Toutefois, ces actions sont laissées en suspend à la fin de l'année 2006. La mise en œuvre de la Loi organique relative aux lois de

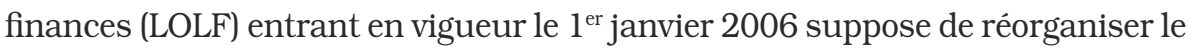
ministère. Un secrétariat général est créé aux côtés d'une direction générale de l'enseignement scolaire (DGESCO). Celui-ci intègre les directions techniques, dont la DEP, qui voit son influence sur le cabinet s'éloigner. La LOLF suppose 
de revoir l'organisation interne de ce qui, cinq mois après, devient la DEPP pour que son dialogue avec la DGESCO soit plus efficace.

Ces aléas politiques (quatre réorganisations de la direction en dix ans) ont pour conséquence directe de rendre incertaine et discontinue la production statistique. Pendant la période Allègre, les statisticiens se concentrent sur l'exploitation des enquêtes en cours et la production de données usuelles sur le fonctionnement du système éducatif. Le nombre de commandes à l'attention des chercheurs est divisé par deux (37 en 1997, 20 en 1998). Le nombre d'évaluations publiées par an passe de 29 en 1997 à 10 en $2000^{39}$. En 1998, la direction perd un appel d'offres pour participer à la conception de l'enquête PISA $^{40}$ lancée par l'Organisation de coopération et de développement économiques (OCDE). Les outils mis en œuvre à la période suivante consistent plus en la synthèse et le perfectionnement d'outils existants qu'en innovations méthodologiques majeures. Les statisticiens refondent les indicateurs de performance des établissements du second degré en 2001-2002 et relancent les évaluations bilans en 2003. Ils diffusent plus largement leurs travaux grâce aux nouvelles technologies (mise en ligne des publications en 2001, mise en ligne de la base ACCADEMIA en avril 2003). Ils proposent de nouvelles publications comme Regards sur le système éducatif (lancé dès 1998) et les Notes d'évaluation (à partir de septembre 2003). Cette entreprise de synthèse et de rationalisation de l'existant est favorisée par les activités du Haut conseil de l'évaluation de l'école et l'organisation en 2004 du débat sur l'avenir de l'école auxquelles les statisticiens sont étroitement associés. Enfin, l'application de la LOLF en 2006 implique de réduire le nombre d'indicateurs existant et d'en produire de nouveaux, donc de repenser leur construction et leur signification.

On retrouve ces aléas dans la composition de la direction qui, globalement, est moins stable que précédemment. La politisation de son activité sous le ministère de Claude Allègre se traduit par une baisse du nombre de personnels INSEE désireux d'y être détachés pour trois ans. Leur proportion passe de $11,6 \%$ en 1998 à 9,3\% en 2001. Les années de reconstruction se traduisent par l'augmentation entre 2001 et 2005 de la part de contractuels (de 12,9\% à $16,4 \%$ ), d'ingénieurs d'étude ou de recherche (de 16,2\% à 19,5\%), d'administrateurs INSEE (dont la proportion remonte à 12,8\% en 2005) et de professeurs

39 Xavier Pons, L'évaluation des politiques éducatives et ses professionnels..., op. cit.

40 Programme international de suivi des acquis des élèves. 
du secondaire (de 8,7\% à 11,1\%), dont l'accroissement est lié à la relance des évaluations bilans des résultats des élèves en fin de troisième. La part de ces différentes catégories diminue légèrement depuis 2005.

Dans un tel contexte d'incertitudes politiques sur le devenir et les missions de la direction, on assiste à une lutte de territoires administratifs qui concrètement se manifeste par la réactivation de multiples clivages. Certains sont anciens comme celui du "Soviet de Vanves" ${ }^{41}$ ou celui entre producteurs et utilisateurs des données. Ce dernier réapparait par exemple avec la gestion de certaines publications. Historiquement, les Notes d'information (NI) sont les plus anciennes, les Notes d'évaluation (NE) datant de 2003. Mais la distinction entre elles, tout comme les critères de publication dans chacune d'elle, ne sont pas clairs aux yeux de nos interlocuteurs. Des personnes n'appartenant pas à la sous-direction de l'évaluation mais produisant une étude originale et utile au pilotage du système éducatif peuvent voir leur travail publié dans une NE. A l'inverse certaines publications des bureaux d'évaluation se font dans des NI. Ce flou est à l'origine d'une nouvelle polémique au sein de la direction entre les producteurs et les utilisateurs de données, les premiers publiant plutôt dans les NI et les seconds dans les NE. Plus globalement, chaque segment professionnel s'étonne de ne pas voir mieux pris en compte ses intérêts. Les administrateurs de l'INSEE font part en entretien de leur étonnement quant au fonctionnement de la direction à leur arrivée, en vase clos et sur le mode de l'implicite, et regrettent de ne pas être plus sensibilisés par leurs nouveaux collègues expérimentés aux enjeux et aux problématiques éducatives du moment. Les incertitudes de la fonction évaluation au ministère réactivent aussi les clivages entre les statisticiens et les évaluateurs. Ces derniers se plaignent de ce qu'il n'existe pas un vrai "métier" d'évaluateur comme il existe un métier de statisticien alors que leur activité nécessite de constamment se remettre en question et d'utiliser des techniques que même les administrateurs INSEE ne maîtrisent pas toujours (comme la psychométrie). Enfin la période est marquée par un clivage politique entre les tenants de l'effet miroir et ceux, nommés plus récemment à des postes de cadres, qui veulent que les statisticiens concourent à la formulation de jugements normatifs sur le fonctionnement du système

41 «Il est resté une culture Vanves qui est restée ancrée chez les gens de l'administration centrale. [...] Je me souviens d'un directeur qui parlait du "Soviet de Vanves". On était les troublions, la chienlit. C'est resté très longtemps. Y compris maintenant quand [le nouveau directeur] est arrivé, des gens se sont étonnés qu'on réagisse peu" (entretien avec un chargé de mission de la DEPP). 
éducatif. Les premiers insistent sur la nécessité de se cantonner à un constat objectif des faits. Les seconds souhaitent que les chargés d'études aident à la décision politique en fournissant un avis éclairé et qu'ils communiquent mieux sur leurs travaux.

\section{Conclusion}

Au total, de 1957 à 2007, selon les impératifs politiques et administratifs du moment, mais aussi les progrès technologiques et méthodologiques, les statisticiens du ministère sont alternativement perçus comme de simples producteurs de données administratives au service de l'action de l'État, des chargés d'études autonomes produisant un savoir le plus scientifique possible ou des experts supposés aider au pilotage du système éducatif. Émergent ainsi, au cours de la période, diverses figures de ce métier qui sont toutes liées à des configurations sociales et historiques spécifiques (les "administrateurs" et "modernisateurs" de la statistique, les "statisticiens de Vanves" et les "statisticiens du miroir") et dont l'affirmation donne naissance à un univers administratif et professionnel fortement clivé. Il reste alors à déterminer dans quelle mesure la statistique scolaire, science d'État relativement ancienne ${ }^{42}$, contribue à façonner des "esprits d'État", donc si sa force performative éventuelle rencontre, et avec quelle efficacité et selon quels processus, les structures cognitives des acteurs du système scolaire, dès lors enclins à accepter cet outil particulier, et politique, de construction et de classement des réalités sociales ${ }^{43}$.

42 Xavier Pons, "L'État éducateur français à travers sa production statistique : configurations historiques d'une science d'État (1797-2007)", in Julien Barroche, Nathalie Le Bouëdec, Xavier Pons (coord.), Figures de l'État éducateur. Pour une approche pluridisciplinaire, Paris, L'Harmattan, 2008, 276 p.

43 Pierre Bourdieu, "Esprits d'État", Actes de la recherche en sciences sociales, vol. 96-97, mars 1993, p. 49-62. 